\title{
CEFALEIA EM SALVAS \\ ASPECTOS CLINICOS E TERAPEUTICOS EM 26 CASOS
}

WILSON LUIZ SANVITO *

CHARLES PETER TILBERY**

A cefaléia-em-salvas é conhecida sob várias denominações eponímicas, além de outras variantes de nomenclatura dando ênfase a um aspecto clínico do quadro 4,5,13,16. Em nosso país, duas são as designações empregadas com maior frequência: cefaléia histamínica e cefaléia de Horton. Nos Estados Unidos da América do Norte, assim como em alguns paises europeus, o nome consagrado para esta afeç̧ão é "cluster headache", sendo cefaléia-em-salvas uma tradução livre desta expressão alienigena ${ }^{13}$.

Este tipo de cefaléia, que evolui em surtos, costuma ter inicio nos individuos de meia-idade. Predomina no sexo masculino numa proporção que chega até 6:111. A cefaléia caracteriza-se por ser paroxística, unilateral (comumente localizada nas regiões temporal e ocular) e de grande intensidade. A dor, que rapidamente atinge o seu climax, pode assumir o caráter de queimação, agulhada ou, então, apresentar-se sob a forma perfurante ou dilacarente; às vezes se associa um componente pulsátil. Nem sempre o doente consegue definir o tipo de dor. Por ocasião da crise, se o doente estiver deitado ele se levanta, se põe a caminhar de um lado para outro, inquieto, comprimindo fortemente a região acometida. As crises podem durar 10 minutos a 2 horas e costumam incidir mais de uma vez nas 24 horas, sendo frequente pelo menos uma crise noturna que habitualmente se instala 1 a 2 horas após o adormecimento do individuo. No final da crise a dor se atenua e acaba por desaparecer, podendo, contudo, permanecer um fundo de desconforto por algum tempo na região acometida. Como manifestações associadas são encontradas com frequência lacrimejamento, congestão ocular e rubor na região afetada, além de obstrução nasal uni- ou bilateral. Os vômitos raramente ocorrem. Em alguns casos pode ser observado sindrome de Claude Bernard-Horner, de modo transitório. Os periodos de crise podem durar de 2 a 6 semanas, ou mesmo mais, sendo comuns longos períodos de acalmia (esses períodos intercriticos podem durar até anos). Existem formas crônicas deste tipo de cefaléia, com duração prolongada dos surtos (vários meses). A crise pode ser reproduzida por injeção subcutânea de histamina, porém nem sempre este evento ocorre, circunstância que não permite descartar a hipótese de cefaléia-em-salvas. Outros fatores

\footnotetext{
Trabalho da Disciplina de Neurologia do Departamento de Medicina da Faculdade de Ciências Médicas da Santa Casa de Såo Paulo: - Prof. Pleno de Neurologia; * Prof. Assistente de Neurologia.
} 
desencadeantes das crises são nitroglicerina sublingual e outros medicamentos vasodilatadores periféricos, além da ingestão de bebidas alcoólicas, porém apenas nos períodos suscetiveis.

A grande intensidade da manifestação álgica torna a cefaléia-em-salvas um um quadro dramático, que tem merecido por parte de alguns até a denominação de cefaléia-suicídio. Esta vertente do problema tem preocupado os médicos no sentido de uma terapêutica efetiva na prevenção das crises. Este é o objetivo fundamental deste trabalho.

\section{CASUISTICA E METODOS}

Vinte e sels casos de cefaléla-em-salvas, provenientes de nossa clínica privada e da Santa Casa de Sá Paulo foram diagnosticados e tratados entre 1970 e 1979 (Tabela 1). Os critérios diagnósticos, sempre clínicos (Tabelas 2 e 3), foram de duas ordens: 1) manifestaçరes fundamentais traduzldas por crises de rápida duraçáo (10 minutos a 2 horas), unilateralidade do fenômeno álgico, presença de um ou mals surtos constítuidos por um elenco de crises; 2) manifestaçoes secundárias ou associadas, tendo particular importancia o lacrimejamento, congestáo ocular, obstruçáo nasal e sindrome de Claude Bernard-Horner. Apenas em um caso fol utilizada a prova farmacológica (dinitrato de isosorbitol*), com a finalidade de reproduzir a crise. $O$ tratamento, sempre preventivo, foi realizado com as seguintes drogas e esquemas: 1) maleato de metisergida, em dosagem que vartou de 3 a $6 \mathrm{mg}$ por dia, durante 4 semanas; 2) prednisona, na dose inicial de 40 a $60 \mathrm{mg}$ por día e com reduçáo gradativa da dose até a completa eliminaçăo do medicamento no fim da 44 semana; 3) associaçăo de maleato de metisergida e prednisona, nas doses referidas nos itens 1 e 2, durante 4 semanas. Em pacientes até então năo-tratados, a primeira opçáo fol o uso do maleato de metisergida; a prednisona fol utilizada isoladamente no caso de intolerancia ou resposta nula a metisergida. Finalmente, a associaçăo medicamentosa foi utilizada quando a resposta ao maleato de metisergida era parcial. A prednisona fol introduzida no tratamento de nossos pacientes a partir de 1972. Em pacientes previamente tratados eliminava-se, pelas informaçóes prestadas, as drogas que provocavam intolerancia ou cujo resultado tivesse sido nulo. A resposta aos tratamentos propostos obedeccu aos seguintes critérios: resultado nulo ((K.N.); resultado regular (K.R.), caracterizado pela diminuiçăo do número e intensidade das crises; resultado bom (R.B.), caracterizado pela aboliçăo das crises (Tabela 4).

\section{COMENTARIOS}

A cefaléia-em-salvas é uma variante das cefaléias vasculares, sendo consideravelmente mais rara que a enxaqueca 12,15. E provavelmente a mais dramática e intensa forma de cefaléia que o homem conhece ${ }^{15}$, e frequentemente é confundida com a neuralgia do trigêmeo, sinusite e até com a cefaléia psicogênica 3,12. Daí sua importância clínica.

\footnotetext{
* Isordil (10 mg).
} 


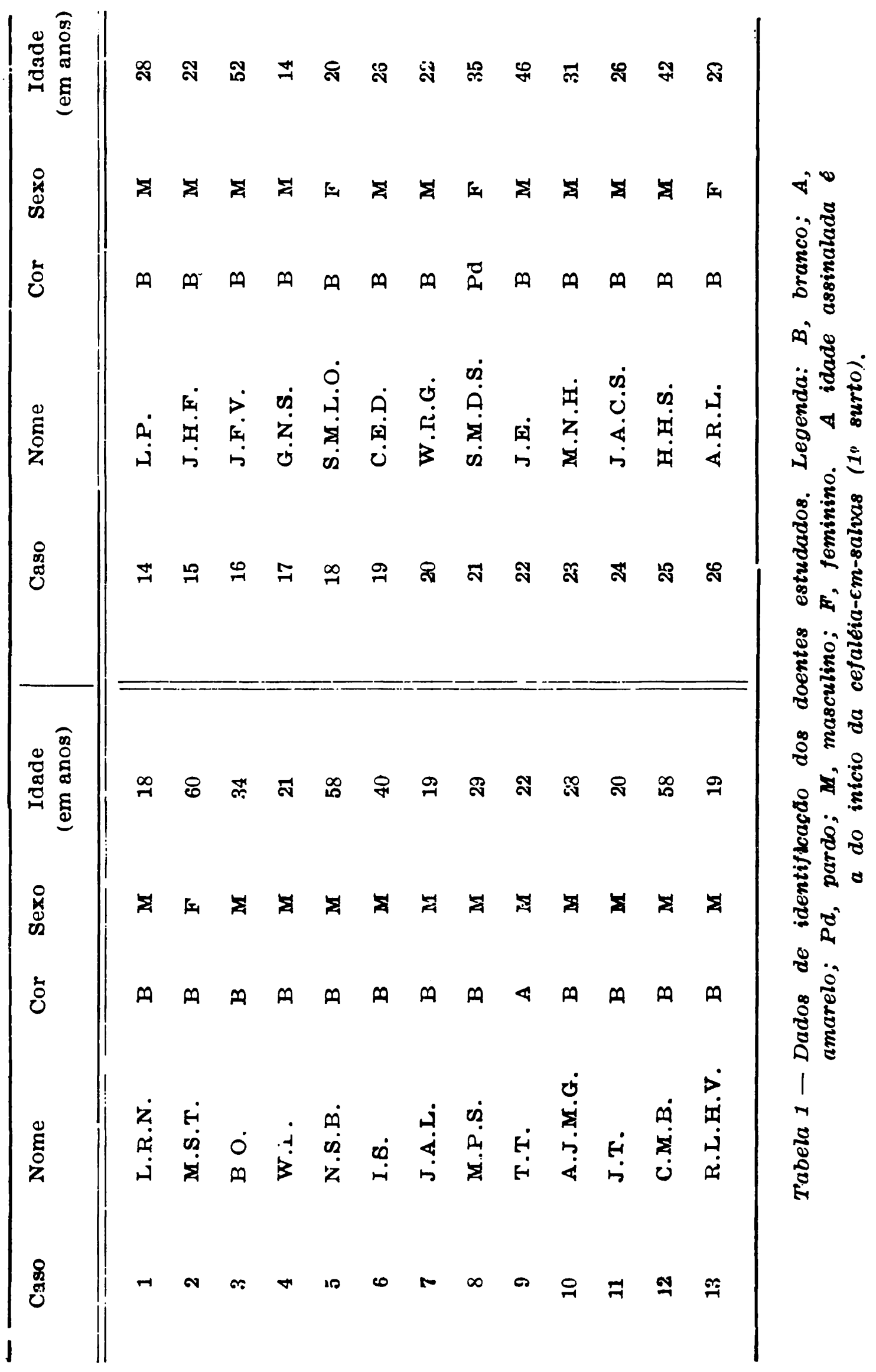




\begin{tabular}{|c|c|c|c|c|c|c|c|}
\hline Caso & $\begin{array}{c}\text { Duraçăo } \\
\text { da crise } \\
\text { (média } \\
\text { em min.) }\end{array}$ & $\begin{array}{c}\text { Crises } \\
\text { diurnas }\end{array}$ & $\begin{array}{c}\text { Crises } \\
\text { noturnas }\end{array}$ & Caso & $\begin{array}{l}\text { Duraçăo } \\
\text { da crise } \\
\text { (média } \\
\text { cm min.) }\end{array}$ & $\begin{array}{l}\text { Crises } \\
\text { diurnas }\end{array}$ & $\begin{array}{c}\text { Crises } \\
\text { noturnas }\end{array}$ \\
\hline 1 & 20 & + & + & 14 & $20-30$ & + & + \\
\hline 2 & 15 & + & + & 15 & * & + & + \\
\hline 3 & 20 & + & - & 16 & $15-20$ & + & + \\
\hline 4 & 20 & + & + & 17 & * & + & - \\
\hline 5 & 30 & + & - & 18 & $30-60$ & + & - \\
\hline 6 & 45 & + & - & 19 & $20-30$ & + & - \\
\hline 7 & 10 & + & + & 20 & $20-30$ & + & - \\
\hline 8 & * & + & - & 21 & $30-60$ & + & - \\
\hline 9 & $40-60$ & + & + & 22 & * & + & - \\
\hline 10 & $15-20$ & + & + & 23 & 30 & + & - \\
\hline 11 & 60 & + & + & 24 & $120-180$ & + & + \\
\hline 12 & 60 & + & - & 25 & 30 & + & - \\
\hline 13 & 60 & + & + & 26 & 20 & - & + \\
\hline
\end{tabular}

Tabela z-Média de duraça e hordíno das crises: " não foi possivel precisar.

Esta forma de cefaléia predomina no sexo masculino, ao contrário da enxaqueca ${ }^{3,11}$, em proporções que variam de 3 a 6 para $13,11,12$. Em nossa casuística a proporção foi de 5:1, sendo a média de idade de 31,5 anos, discretamente mais baixa que a referida na literatura que se situa ao redor dos 39 anos $^{3}$. As idades limites foram 14 e 60 anos, enquanto na casuistica de Lance 21 foram de 8 e 62 anos.

$O$ diagnóstico da cefaléia-em-salvas, eminentemente clínico, se impõe pelo quadro álgico intenso e unilateral, pelo elenco de crises num surto, pela presença de crise(s) noturna(s) (que costuma ocorrer 90 a 120 minutos após a conciliação do sono ou após cochilo vespertino) e pela presença das manifestações associadas (principalmente de expressão neurovegetativa). Em 69,2\% de nossos casos ocorreram manifestações associadas (Tabela 3), dados que a grosso modo, correspondem aos da literatura. Os principais fatores desencadeantes da cefaléia-em-salvas são as bebidas alcoólicas, as drogas vasodilatadoras (histamina, nitroglicerina) e a fase REM do sono. Em nossa casuística, a crise foi desencadeada pela ingestão de bebidas alcoólicas em 7 casos e em um caso (caso 18) 


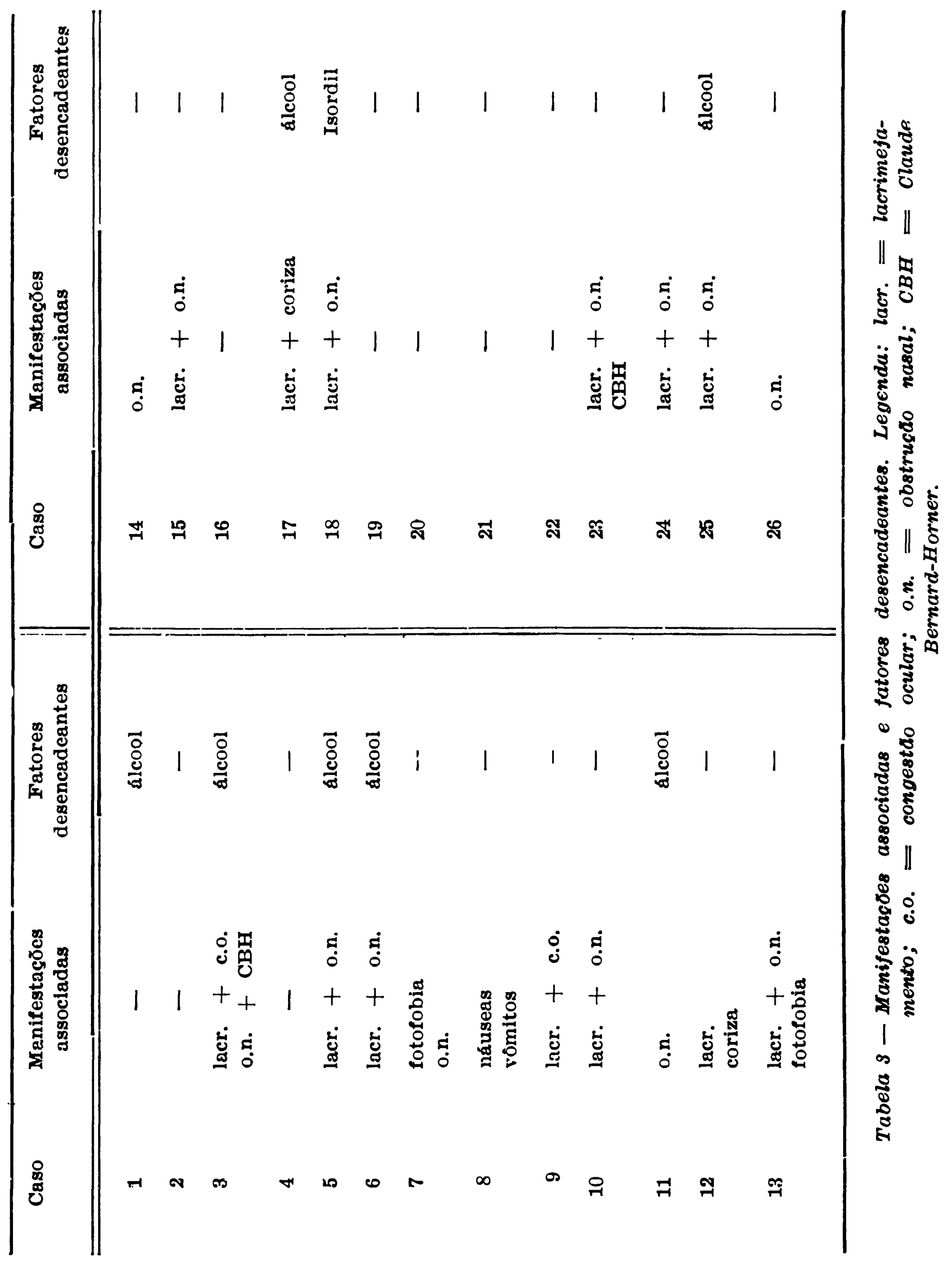




\begin{tabular}{|c|c|c|c|c|c|c|c|c|c|}
\hline \multirow{2}{*}{ Caso } & \multicolumn{3}{|c|}{ Esquema terapêutico } & \multirow{2}{*}{ Result. } & \multirow{2}{*}{ Caso } & \multicolumn{3}{|c|}{ Esquema terapêutico } & \multirow{2}{*}{ Result. } \\
\hline & $\mathbf{M} . \mathbf{M}$ & Pred. & Assoc. & & & M.M. & Pred. & Assoc. & \\
\hline 1 & $\mathbf{x}$ & - & + & B & 14 & 0 & 0 & + & B \\
\hline 2 & + & 0 & - & $\mathbf{B}$ & 15 & + & - & - & $\mathbf{B}$ \\
\hline 3 & $\mathbf{x}$ & - & $\mathbf{x}$ & $\mathbf{R}$ & 16 & 0 & - & $\mathbf{x}$ & $\mathbf{R}$ \\
\hline 4 & - & - & + & B & 17 & + & - & - & $\mathbf{B}$ \\
\hline 5 & + & - & - & B & 18 & 0 & + & - & $\mathbf{B}$ \\
\hline 6 & + & - & - & $\mathbf{B}$ & 19 & 0 & - & $\mathbf{x}$ & $\mathbf{R}$ \\
\hline 7 & 0 & - & $\mathbf{x}$ & $\mathbf{R}$ & 20 & $\mathbf{x}$ & - & - & $\mathbf{R}$ \\
\hline 8 & 0 & $\mathbf{x}$ & - & $\mathbf{R}$ & 21 & - & + & - & B \\
\hline 9 & $\mathbf{x}$ & 0 & - & $\mathbf{R}$ & 22 & + & - & - & $\mathbf{B}$ \\
\hline 10 & 0 & - & 0 & $\mathbf{N}$ & 23 & 0 & - & $\mathbf{x}$ & $\mathbf{R}$ \\
\hline 11 & 0 & + & - & B & 24 & 0 & - & 0 & $\mathbf{N}$ \\
\hline 12 & 0 & - & - & $\mathbf{N}$ & 25 & - & - & + & B \\
\hline 13 & + & - & - & B & 26 & 0 & + & - & $\mathbf{B}$ \\
\hline
\end{tabular}

Tabela 4 - Esquemas terapeuticos empregados. Legenda: M.M. = maleato de metisergida; Pred. = prednisona; As8oc. = associacto; Result. = resultado; $\boldsymbol{X}=$ droga usada com resposta parcial; $+=$ droga usada com resposta total; $0=$ droga usada sem resposta; $-=$ droga nao usada; $B=$ bom; $R=$ regular; $N=$ nulo.

a crise foi reproduzida, com fins diagnósticos, pelo uso do dinitrato de isosorbitol (Tabela 3). Em 50\% de nossos pacientes ocorreram crises noturnas, manifestação que sugere o sono REM como fator desencadeante; em apenas um paciente as crises eram exclusivamente noturnas (caso 26).

Kudrow ${ }^{11}$ classifica a cefaléia-em-salvas em três formas clínicas: periódica, crônica e atípica. Todos os nossos casos podem ser enquadrados dentro da forma periódica, com exceção do caso 24 que apresentou uma forma crônica. Esta forma crônica tem como característica essencial a longa duração do surto, praticamente sem períodos de remissão, e a sua caracterização é importante para a conduta terapêutica.

Aspecto clínico curioso da cefaléia-em-salvas é a regularidade da duração dos surtos em determinados pacientes. Em nossos casos 13 e 16 a duração dos surtos é de 3 e 4 semanas respectivamente. Sob este aspecto poderiamos denominar a estas formas de cefaléia-calendário.

A terapêutica da cefaléia-em-salvas é muito controvertida e numerosas drogas têm sido ensaiadas nos últimos anos: metisergida ${ }^{6}$, prednisona ${ }^{9}$, propranolol ${ }^{14}$, 
pizotifen 2,4. Talvez a grande dificuldade para a avaliação correta da eficácia da droga em uso, resida na forma efêmera deste tipo de cefaléia na maior parte dos casos. Alguns autores ${ }^{1,17}$ acreditam que a fisiopatologia da cefaléia depende de vasodilatação das artérias carótidas, interna e externa. Baseados na presença de receptores ${ }^{\mathrm{H} 1}$ e ${ }_{\mathrm{H} 2}$ na carótida interna e de receptores ${ }^{\mathrm{H} 2}$. na carótida externa, Anthony e col. dosaram os niveis de histamina em 49 doentes antes, durante e depois de uma crise e notaram aumento estatisticamente significante dos níveis histamínicos em 45 casos. Em seguida bloquearam os receptores ${ }^{\mathrm{H} 1}$ com mepyramina em 10 doentes e os receptores $\mathrm{H} 2$ com cimetidine em 15 doentes. Em ambos os grupos não houve resposta estatisticamente significante. Verger e col.17 empregaram em 10 doentes a cimetidine na dose de $400 \mathrm{mg}$ diários, por um período de 6 semanas, e também não obtiveram resultados. Um dos doentes de nossa investigação (caso 12) sofria de úlcera gástrica e na vigência de cimetidine nos procurou por crises de cefaléia-em-salvas. A propósito, alguns autores ${ }^{3,12}$ relatam a concomitância de úlcera péptica e cefaléia-em-salvas e procuram justificá-la pelo aumento do tono vagal durante as crises de cefaléia. Por outro lado, Horton 8 cita um caso em que a dessensibilização histamínica melhorou a cefaléia e a úlcera péptica associada.

Giampaoli 7 utilizou a epinefrina em 7 casos de cefaléia-em-salvas, baseado na teoria da vasodilatação do sistema carotídeo como determinante da cefaléia, obtendo excelente resposta. Esta eficácia não tem sido comprovada por outros autores. Espadaler e col.4 e Ekbom ${ }^{2}$ utilizaram o pizotifen (BC-105) com resultados favoráveis em muitos casos, porém esta droga não parece ser a ideal. Jammes ${ }^{9}$ relata bons resultados com o uso da prednisona, pois muitos doentes que inicialmente respondiam à metisergida, ergotamina, propranolol e outras drogas, ulteriormente se tornavam refratários a essas drogas. Foram estudados por Jammes 19 pacientes que utilizaram $30 \mathrm{mg}$ de prednisona no 19

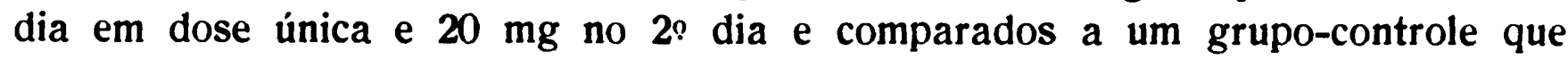
utilizou placebo. Houve diferença significativa entre os dois grupos com melhora acentuada em 17 dos 19 doentes em uso da droga ativa, sendo que o esquema terapêutico empregado manteve 14 pacientes assintomáticos por um periodo de 60 dias. Sempre que necessário, por recrudescimento da dor, era utilizada dose única de $30 \mathrm{mg}$ de prednisona com bom resultado. Horton (cit. por Jammes ${ }^{9}$ ) acreditava que a cefaléia-em-salvas estaria vinculada à sensibilidade histamínica, condição que justificaria o sucesso da prednisona.

Friedman e Elkind ${ }^{\circ}$ utilizaram a metisergida na dose de 6 a $16 \mathrm{mg}$ por dia em 54 indivíduos com cefaléia-em-salvas, obtendo melhora em 20 casos. Em 39\% dos casos tratados com metisergida ocorreram efeitos colaterais importantes como fraqueza, tontura, precordialgia, parestesias nos membros, artralgias e outros, havendo necessidade de suspender a droga em muitos casos. Kudrow ${ }^{10}$ utiliza como primeira opção a metisergida, porém comenta que a fibrose retroperitoneal, o potente efeito vasoconstrictor e à contraindicação desta droga em várias doenças limitam o seu uso. Este autor se pauta pela seguinte conduta: utiliza, inicialmente, a metisergida na dose de $8 \mathrm{mg}$ por dia por um periodo de 21 dias; não ocorrendo resposta em 10 dias, utiliza a prednisona na dose de $40 \mathrm{mg}$ por dia, com redução gradativa da droga até a sua completa suspensão 
ao atingir o 210 dia. Em 41 casos de cefaléia-em-salvas, de um grupo de 77; houve melhora em $75 \%$ com a metisergida. A prednisona foi utilizada em. outro grupo de 77 casos, havendo resposta em 59. Por este estudo Kudrow. conclui que a prednisona é superior à metisergida. Não havendo resposta a estas drogas, o autor utiliza o carbonato de lítio na dose de $600 \mathrm{mg}$ por dia durante 3 semanas (se necessário ele emprega $900 \mathrm{mg} /$ dia). Com este último esquema, o autor refere bons resultados em $87 \%$ dos casos.

Em nossa investigação utilizamos o maleato de metisergida em 20 casos numa dosagem que variou de 3 a $6 \mathrm{mg}$ por dia, durante 4 semanas. Em 7 casos o resultado foi bom, regular em 2 e nulo em 9 (Tabela 4). Em dois casos não houve tolerância ao medicamento (casos 12 e 18). A porcentagem de melhora, com esta droga, em nossa casuistica é de 50\% (não considerando os pacientes que apresentaram intolerância) e está abaixo daquela de Kudrow; contudo cumpre ressaltar que as doses utilizadas por nós foram mais baixas. $O$ uso isolado da prednisona foi tentado em 8 casos, sendo o resultado bom em 4 casos, regular em 1 e nulo em 3. O esquema misto (metisergida+ +prednisona) foi utilizado em 11 casos, com 4 resultados bons, 5 regulares e 2 nulos. Numa avaliação global pudemos concluir que os resultados forain satisfatórios. Assim, 15 pacientes ficaram livres de suas crises $(57,6 \%), 8$ apresentaram melhora significativa $(30,7 \%)$ e em 3 não houve resposta. Em outras palavras, $88,3 \%$ dos casos apresentaram algum tipo de resultado (bom ou regular) ao tratamento instituido. Quanto aos resultados nulos (casos 10, 12 e 24), deve-se ressaltar que um paciente (caso 12) não tolerou a metisergida e por ser portador de úlcera péptica hemorrágica não pôde se submeter ao tratamento com prednisona. Outro paciente com resultado nulo (caso 24) apresentou a forma crônica da cefaléia-em-salvas, indicação precisa, segundo Kudrow 1n,11, para o uso dos sais de lítio.

\section{RESUMO}

Vinte e seis casos de cefaléia-em-salvas são estudados do ponto de vista clínico e terapêutico. Nos aspectos clínicos são ressaltados a dramaticidade deste tipo de cefaléia, o nítido predomínio no sexo masculino, a média de idade dos pacientes, a presença de manifestações associadas e os fatores desencadeantes das crises nos periodos suscetíveis. Do ponto de vista terapêutico três esquemas medicamentosos foram utilizados: maleato de metisergida, prednisona e associação de ambas as drogas. Em 88,3\% dos casos um resultado significativo foi alcançado, traduzido por melhora ou abolição das crises.

\section{SUMMARY}

\section{Cluster headache: clinical and therapeutic aspects in 26 cases.}

The clinical and therapeutic aspects in 26 patients with cluster headache are reported. The patient's age ranged between 14 and 60 years old (average 31.5 years); 22 were male and 4 female. The patients constitued a consecutive 
series of typical cases (only one was a chronic cluster) with regular headache attacks; in 69.2 per cent of the cases there were associated symptoms and signs (ipsilateral lacrimation, stuffiness of the ipsilateral nostril and Horner's syndrome). In seven patients the cluster was induced by alcohol ingestion (during an active cluster period) and a cluster attack was reproduced by isosorbitol dinitrate (vasodilatador drug) in one patient. All patients received orally either methysergide maleate, prednisone or both. The first drug used, in 20 patients, was methysergide maleate in a dosage of 3-6 $\mathrm{mg}$ daily over a four weeks period. Eight patients received prednisone only, which presented side-effects or no improvement with methysergide, in a dosage of $40-60 \mathrm{mg}$ daily and tapered off over a period of four weeks. Methysergide maleate and prednisone were given to 11 patients in association, because of moderate effect of the first drug. The clinical effect of the treatment was judged according to the following three categories: freedom from the attacks; slight improvement of the attacks; no improvement of the attacks. In 57.6 per cent of the cases (15 patients) there was freedom of the attacks, in 30.7 per cent of the cases ( 8 patients) there was a slight improvement and in 3 patients there was no improvement.

\section{REFERENCIAS}

1. ANTHONY, M.; LANCE, J. W. \& LORD, G. - Migrainous neuralgia: blood histamine levels and clinical response to $H 1$ and $H_{2}$ receptor blockade. International Migraine Symposium, London, September 1976.

2. EKBOM, K. - Prophylactic treatment of cluster headache with a new serotonin antagonist, BC 105. Acta Neurol. Scand. 45:601, 1969.

3. EKBOM, K. - Clinical aspects of cluster healache. Headache 13:176, 1974.

4. ESPADALER, J. M.; GIMENO, A. \& LAGE, A. M. - A cooperative study on clinical aspects of migraine and vascular headache and their response to Sandomigran. Arch. Neurobiol. (Madrid) 37 (Suppl) :206, 1974.

5. FARIAS DA sILVA, W. - Consideraçסes sobre a enxaqueca e a sindrome de Horton. Neurobiologia (Recife) 2:171, 1974.

6. FRIEDMAN, A. P. \& ELKIND, A. H. - Appraisal of methysergide in treatment of vascular headaches of migraine type. JAMA 184:125, 1963.

7. GIAMPAOLI, A. V. - Cluster headaches: a new treatment. West. J. Med. 120:326, 1974.

8. HORTON, B. T. - Histaminic cephalalgia. JAMA 160:468, 1956.

9. JAMMES, J. L. - The treatment of cluster headaches with prednisone. Dis. Nerv. Syst. $36: 375,1975$.

10. KUDROW, L. - Comparative results of prednisone, methysergide and lithium in cluster headache. In Green, R. - Current Concepts in Migraine Research. Raven Press. New York, 1978, pp. 159-163. 
11. KUDROW, L. - Cluster headache: diagnosis and management. Headache 19:142, 1979.

12. LaNCE, J. W. - Cefaléia. Versăo brasileira. Manole, Săo Paulo, 1976, pp. 161-177.

13. RAFFAeLLI, Jr., E. - Cefaléias crônicas e enxaqueca. Ars. Curandi 12:5, 1979.

14. RYAN, R. E. - Modern concepts of the management of histaminic cephalalgia. South Med. J. $56: 1384,1963$.

15. RYAN, R. E. \& RYAN, Jr., R. E. - Headache and Head Pain. Mosby, Saint Louis, 1978, pp. 176207.

16. Sanvito, W. L. - Síndromes Neurológicas. Manole, Săo Paulo, 1977, pp. 188-190.

17. VEGER, T.; RUSSEL, D. \& SJAASTAD, O. - Histamine H2 antagonists and cluster headache (letter). British Med. J. 2:585, 1976.

Disciplina de Neurologia, Departamento de Medicina - Fraculdade de Ciencias Médicas da Santa Casa de Säo Paulo - Rua Cesdrio Motta Ji. 112 01821 São Paulo, SP - Brasil. 